\title{
EDITORIAL
}

\section{L'ARODF, une société savante et responsable}

\section{Guy Bounoure}

Président du Conseil d'administration de I'ARODF
Adresse de correspondance orthoalbi@wanadoo.fr
'Association de la Revue d'Orthopédie dento-faciale (ARODF), en charge de la publication de Lla Revue d'ODF, a été reconnue " société savante " le 12 février 2021. Elle est répertoriée comme telle depuis, par les instances habilitées à faire connaître cette qualité, notamment aux sociétés scientifiques.

Cette reconnaissance vient saluer la qualité des travaux publiés trimestriellement dans la Revue d'ODF depuis plus de 50 ans et dans toutes les disciplines qui constituent le corps de notre spécialité.

Cette qualification vient encore rappeler que, dès sa fondation, les missions statutaires de l'Association prévoyaient de mettre en place puis d'entretenir l'essor de la formation continue des praticiens de la spécialité, et ce, 15 ans avant l'avènement officiel de l'orthopédie dento-faciale comme spécialité.

Les efforts et la persévérance de l'ensemble des rédacteurs et des auteurs ont permis de participer à la formation continue, tant aux plans clinique que fondamental, en accueillant les articles originaux de spécialistes étrangers ou en effectuant des échanges de publication afin de diffuser, dans le cadre européen des publications sœurs, des recherches sur des thèmes innovants, voire peu développés dans la littérature.

Les six articles du précédent numéro de la Revue et les quatre de ce numéro, tous consacrés aux classes II subdivision, avec Yves Soyer pour rédacteur en chef des deux numéros, constituent un parfait exemple de synthèse complète mais diversifiée sur un thème qui mérite aujourd'hui une mise à jour de nos connaissances.

Peu après I'obtention de cette reconnaissance, I'instance dirigeante de I'Association de la Revue d'ODF a souhaité devenir membre du Conseil National Professionnel (CNP) ODF-ODMF, qu'elle a intégré récemment - le 6 juillet 2021 - à la suite d'un vote favorable du Conseil d'administration du CNP ODF-ODMF.

\section{Qu'est-ce que le CNP ODF-ODMF ?}

Ce CNP est organisé selon un cadre réglementaire strict, issu du décret du 9 janvier 2019 qui fixe les missions, la composition et le fonctionnement des différents Conseils Nationaux Professionnels. 
Après intégration de I'ARODF, le CNP ODF-ODMF sera composé de huit membres :

- 3 sociétés savantes : la Société Française d'Orthopédie Dento-Faciale, la Société Médicale d'Orthopédie Dento-Maxillo-Faciale, I'Association de la Revue d'Orthopédie Dento-Faciale,

- 2 collèges représentant les universitaires : le Collège des Enseignants en Orthopédie DentoFaciale, le Collège National des Enseignants en Chirurgie Maxillo-Faciale, Stomatologie et Chirurgie Orale,

- enfin, 3 syndicats professionnels : le Syndicat Français des Spécialistes en Orthodontie, le Syndicat des Orthodontistes de France, le Syndicat Français des Médecins Orthodontistes.

Ainsi, le CNP ODF-ODMF regroupe donc " les sociétés savantes et les organismes professionnels de la spécialité en assurant une représentation des différents modes d'exercice ". Les missions des différents CNP sont essentielles, en particulier pour toutes les professions de santé. Elles sont de 5 ordres :

- dans le cadre du développement professionnel continu (DPC), le CNP ODF-ODMF propose " pour la spécialité qu'il représente, des orientations prioritaires, un parcours pluriannuel de DPC et un document permettant à chaque professionnel de retracer les actions de DPC réalisées» (cf. Mise en œuvre du décret 2019-17 du 9 janvier 2019) ;

- le CNP apporte, en outre, " une expertise dans les domaines scientifique et opérationnel » quant à l'organisation et à l'exercice de la spécialité ;

- il « contribue à analyser l'évolution des métiers et des compétences des professionnels de santé " ;

- il peut " participer à des registres épidémiologiques sur la surveillance des événements de santé ou à des registres professionnels d'observation des pratiques " ;

- enfin, "il désigne les représentants de la spécialité pour siéger dans les structures... qui émettent des avis sur les demandes d'autorisation d'exercice ou de reconnaissance des qualifications professionnelles $"$.

Le Conseil national de l'Ordre des chirurgiens-dentistes ainsi que le Conseil national de l'Ordre des médecins, et le Conseil national des Universités peuvent siéger au sein du Conseil d'administration du CNP, chacun avec une voix consultative.

On le voit : par le décret du 9 janvier 2019, la mise en place des CNP marque une étape cruciale dans I'organisation de notre exercice professionnel, face aux obligations auxquelles la spécialité ODF devra se soumettre, selon les modalités d'application du décret.

Pour suivre de près le dossier CNP ODF-ODMF, le Conseil d'administration de I'ARODF a choisi de faire acte de candidature et l'Association, reconnue société savante, a été accueillie pour siéger parmi les sociétés membres qui sont à l'origine de la création de ce CNP spécialisé.

Dans ses domaines d'activités et de compétences, I'ARODF poursuivra les objectifs statutaires qu'elle s'est donnés pour optimiser la qualité et la sécurité des soins orthodontiques et, dans le

NDLR :

Les opinions émises n'engagent que leurs auteurs. cadre des politiques de santé, elle continuera à enrichir les connaissances des professionnels de notre spécialité.

L'ARODF est partie prenante dans la formation continue. 